Meta

Journal des traducteurs

Translators' Journal

\title{
Fonctions et vie des sigles et acronymes en contextes de langues anglaise et française de spécialité
}

\section{Jacqueline Percebois}

Volume 46, numéro 4, décembre 2001

URI : https://id.erudit.org/iderudit/003821ar

DOI : https://doi.org/10.7202/003821ar

Aller au sommaire du numéro

Éditeur(s)

Les Presses de l'Université de Montréal

ISSN

0026-0452 (imprimé)

1492-1421 (numérique)

Découvrir la revue

Citer cet article

Percebois, J. (2001). Fonctions et vie des sigles et acronymes en contextes de langues anglaise et française de spécialité. Meta, 46(4), 627-645.

https://doi.org/10.7202/003821ar
Résumé de l'article

Cet article s'attache à étudier les fonctions et la vie des sigles et des acronymes dans les langues anglaise et française de spécialité en général, et en particulier dans le contexte de l'économie internationale. Il met en évidence une fonction commune, la Fonction Dénominative Lapidaire, qui prendra, selon les cas, un aspect Véhiculaire, Grégaire ou Euphémique. L'observation de la vie des sigles et acronymes met en évidence des variations synchroniques et diachroniques dans leur emploi et celui de leur développement. En conclusion, l'article souligne que, par leurs fonctions, ces néologismes sont un outil linguistique essentiel dans les langues française et anglaise de spécialité. 


\title{
Fonctions et vie des sigles et acronymes en contextes de langues anglaise et française de spécialité
}

\author{
JACQUELINE PERCEBOIS \\ Université Montpellier I, Montpellier, France
}

\begin{abstract}
RÉSUMÉ
Cet article s'attache à étudier les fonctions et la vie des sigles et des acronymes dans les langues anglaise et française de spécialité en général, et en particulier dans le contexte de l'économie internationale. II met en évidence une fonction commune, la Fonction Dénominative Lapidaire, qui prendra, selon les cas, un aspect Véhiculaire, Grégaire ou Euphémique. L'observation de la vie des sigles et acronymes met en évidence des variations synchroniques et diachroniques dans leur emploi et celui de leur développement. En conclusion, l'article souligne que, par leurs fonctions, ces néologismes sont un outil linguistique essentiel dans les langues française et anglaise de spécialité.
\end{abstract}

\begin{abstract}
This paper intends to study the functions and life of initialisms and acronyms in the English and French Languages for Specific Purposes in general, and in particular within the context of international economics. It lays stress on a common function, the Lapidary Denominative Function, and determines under which circumstances this function will assume a Vehicular, Gregarious or Euphemistic aspect. Synchronic and diachronic variations appear in the use of initialisms and acronyms, and the use of the lexical items they result from. As a conclusion, the paper argues that, owing to their functions, these neologisms are an essential linguistic tool in the English and French LSPs.
\end{abstract}

\section{MOTS-CLÉS/KEYWORDS}

sigles et acronymes, variations synchroniques et diachroniques, langues anglaise et française de spécialité, économie du développement

Depuis une cinquantaine d'années s'observe un recours croissant aux sigles et aux acronymes, dans tous les milieux et dans tous les types de discours, à l'écrit comme à l'oral, les linguistes ayant observé la plus grande fréquence d'emploi dans les langues de spécialité (Lerat 1995: 58). Un sigle est épelé; il s'agit d'une initiale ou d'une suite d'initiales servant d'abréviation tandis qu'un acronyme est composé d'un ensemble de lettres ou syllabes initiales, prononcé comme un mot ordinaire. Toutefois, l'acronymie peut être considérée comme un sous-ensemble du processus de réduction du signifiant appelé «siglaison». Le terme «sigles» pourra donc être employé dans un sens général incluant les acronymes. Ces caractéristiques se retrouvent dans la terminologie anglaise : 


\begin{abstract}
Siglaison: initialing.
Sigles et acronymes: Abbreviations, initial-words (Partridge, 1973), initialisms, initial-letter abbreviations (Swan, 1995).

Acronymes, qui sont lus comme des mots: en anglais, acronyms, acronyms or syllable words (Gramley-Patzold, 1992: 27). En 1975, Guilbert (245) signalait les nombreux synonymes d'acronyme: mot-valise, mot-portemanteau, mot-centaure, acronyme ayant été préféré car il pouvait s'intégrer dans une série existante (homonyme, paronyme, etc).

Sigles seuls, qui sont épelés: initial-words, initialisms, initials, abbreviations or letter words (Gramley-Patzold, 1992: 27).
\end{abstract}

Dans cette recherche, nous nous intéresserons aux fonctions et à la vie des sigles et des acronymes. Ces thèmes sont au carrefour de la linguistique, de la sociolexicologie - qui, en sociolinguistique, étudie les rapports entre les phénomènes sociaux et le lexique (Tournier 1991: 168) — et de la psycholinguistique qui étudie les structures et processus mentaux impliqués dans l'acquisition et l'usage de la langue (Widdowson 1996: 130).

Nous étudierons d'abord les fonctions des sigles et acronymes, expressions d'un phénomène de créativité lexicale sans précédent. Dans une deuxième partie consacrée à la vie des sigles et acronymes, nous nous attacherons à étudier les aspects synchroniques et diachroniques de leur évolution ainsi que les conditions de leur succès. L'étude des fonctions et de la vie des sigles et acronymes nous amènera à évoquer certains de leurs domaines d'implantation, pour l'essentiel dans le cadre de la terminologie de l'économie internationale. La collocation «sigles et acronymes» devenant très fréquente dans notre article, nous la désignerons parfois par l'abréviation S\&A. Les exemples cités n'étant pas nécessairement suivis de leur développement dans le corps de cet exposé, nous établirons en annexe une liste des sigles et acronymes suivis de leur développement.

\title{
I. FONCTIONS DES SIGLES ET ACRONYMES
}

Sigles et acronymes se sont implantés dans tous les domaines d'activité, toutes les spécialités. En 1957, dans l'avant-propos de la $2^{\mathrm{e}}$ édition de leur Grand Dictionnaire d'américanismes, Étienne et Simone Deak signalaient la présence de sigles en American

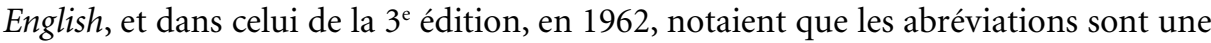
des caractéristiques de la langue américaine, jouant un rôle important dans un pays dont le nom lui-même s'exprime généralement par un sigle: U.S.A. La tendance à la siglaison pourra d'ailleurs se voir attribuée à la pression du système anglo-américain (Rey-Debove, 1998: 191).

Au niveau international, les sigles et les acronymes ont été employés pour donner une dénomination aux institutions et organismes internationaux FMI, OMC, OIT, ainsi qu'aux accords internationaux: GATT, TRIPS (Trade Related Aspects of Intellectual Property Rights). Ce phénomène est particulièrement prononcé dans le commerce international où les économistes Krugman et Obstfeld écrivent en 1997 : «Welcome to the bureaucratic world of trade policy, where everything has a threeletter symbol» tandis que The Economist observe, le 10 octobre 1998 (p. 46), que: «Wherever trade goes, acronyms are sure to follow.» 
Au niveau national, sigles et acronymes baptisent les institutions et organismes nationaux: LSE (London Stock Echange), NYSE (New York Stock Exchange); les organisations administratives telles que l'IRS (Internal Revenue Service); les sociétés commerciales et industrielles telles qu'IBM ou ITT; les universités comme l'UCLA et des diplômes (MBA); les partis politiques, les syndicats... Aucun secteur ne leur échappe.

Quelles sont les fonctions des sigles et acronymes dans la langue? Quelles fonctions justifient leur prolifération? Les concepts de dénotation/connotation et motivation/démotivation jouent un rôle important dans ces fonctions. D’une part, la dénotation d'un mot c'est son sens proprement dit, objectif et stable, la connotation est ce qu'il y a de variable dans le signifié, qui relève d'éléments subjectifs ou du contexte. D'autre part, le sigle joue avec la motivation-démotivation des termes. La motivation est la conscience du lien entre le signifiant et le signifié. La démotivation est la disparition, généralement progressive, de la motivation d'un mot, du fait de l'évolution graphique et phonétique (Tournier 1991: 135); c'est, en l'occurrence, le passage d'une formation syntaxique composée à un sigle épelé ou lu comme un mot.

Nous identifierons une première fonction générale, commune à tous les sigles et acronymes, la Fonction Dénominative Lapidaire (FDL) et nous distinguerons ensuite des FDL véhiculaire, grégaire et euphémique. Selon Calvet (1999: 85), toutes les formes linguistiques que nous utilisons se répartissent sur un vaste éventail de fonctions entre deux pôles: le pôle véhiculaire pour les formes linguistiques destinées à élargir la communication au plus grand nombre, et le pôle grégaire pour les formes linguistiques destinées à limiter la communication au plus petit nombre. Ainsi, par leur fonction véhiculaire, sigles et acronymes pourront servir la compréhension et la diffusion d'un concept, et par leur fonction grégaire, crypter un signifié, l'objectif étant, selon les cas, par la simplification en un syntagme, de faire accéder le plus grand nombre à cet emploi, ou bien par le cryptage, de réserver cet usage à un petit nombre. En revanche, nous nous démarquerons de la position théorique de Calvet en isolant des fonctions véhiculaire et grégaire une fonction euphémique des sigles et des acronymes.

\section{I.1 Fonction Dénominative Lapidaire (FDL)}

Le sigle est un procédé néologique qui a d'abord une fonction dénominative. Sigles et acronymes facilitent la communication en synthétisant le concept considéré. Cette synthétisation est obtenue par la réduction graphique et phonétique de la séquence syntaxique exprimant le concept. La séquence syntaxique est ainsi ramenée à l'unité lexicale type du mot unique. Si le processus de fabrication commence par la réduction graphique, c'est-à-dire la manipulation du signifiant écrit des mots formant une unité sémantique, la réalisation orale du sigle joue un rôle dans cette fabrication (Mortureux, 1997: 54). En outre, la recherche s'oriente vers des sigles ou acronymes lexicalisables, si possible en plusieurs langues, pour ce qui concerne les services de terminologie des institutions et organismes internationaux. La désignation des programmes communautaires que nous évoquerons ultérieurement dans cet article en est un exemple.

D’après Littré, l'origine de nos sigles, ce sont les lettres initiales employées comme signes abréviatifs sur les monuments, les médailles et dans les manuscrits 
anciens. "C'est ce que les Romains appelaient litterae singulae, dont ils ont fait par abréviation siglae et nous sigles. » L'adjectif «lapidaire» signifie: "qui évoque par sa concision et sa vigueur le style des inscriptions sur pierre» (Petit Robert 1999). Nous le choisissons donc délibérément pour rappeler l'origine latine du terme «sigles».

En 1975, Grévisse note que la langue parlée résiste naturellement aux mots trop longs en les abrégeant, reconnaissant là une tendance à économiser l'effort, existant depuis les origines mêmes du français, qui s'est accentuée depuis la fin du XIX ${ }^{\mathrm{e}}$ siècle (1975:113). Grévisse y voit un mode d'abréviation par sigles surtout professionnel, politique ou administratif. Pour lui, il s'agit d'un procédé ou d'un mode de création de mots nouveaux (75-76) et non d'une mode, ce qui va dans le sens de nos observations. La siglaison permet une économie rédactionnelle, c'est-à-dire un gain de temps et d'espace dans la prise de notes, la rédaction d'un article, mais aussi un gain de temps dans l'oralisation des concepts. C'est une des figures de l'économie linguistique, la siglaison pouvant être présentée comme la recherche d'un moindre effort qui se paie toutefois en coût mémoriel (Tournier 1993: 139). Comme exemple de réduction sensible du développement, citons, en Grande-Bretagne, la récente apparition du néologisme Quangos, c'est-à-dire quasi-NGOs, ou quasi Non-Governmental Groups, organisations non gouvernementales quasi autonomes (Malaurie 1999: 2223). Cet exemple offre un cas de "piggy-backing» acronymique, un sigle, NGOs, se trouvant à l'intérieur de l'acronyme Quangos.

La FDL doit satisfaire un impératif d'euphonie. En linguistique, l'euphonie est le résultat harmonieux de la combinaison des sons dans le mot ou la phrase. Or, dans la genèse des sigles et acronymes intervient la lexicoplasie, c'est-à-dire «l'ensemble des artifices auxquels on peut avoir recours pour modeler un signifiant de telle sorte qu'il soit à la fois conforme à la contrainte morpho-phonique et plus facile à mémoriser» (Tournier 1991: 109). Dans la recherche d'euphonie, la constitution d'un acronyme pourra, par exemple, s'accompagner d'ellipse - les initiales de certains des termes qui constituent l'acronyme étant exclues — ou bien certaines initiales de connecteurs tels que prépositions ou conjonctions étant incluses, afin de faciliter la prononciation (Nkwenti-Sager 1990: 97). Ainsi, TRIPS, Trade Related Aspects of Intellectual Property Rights implique l'ellipse du «a » de aspects et $\mathrm{du}$ « $\mathrm{r}$ » de rights, tandis que PANDORA, Prototyping A Navigation Database of Road-Network Attributes (projet du programme européen DRIVE 1), inclut l'article $A$ et la préposition of pour assurer la lisibilité de l'acronyme en un nom chargé de connotations mythiques.

Pour cette raison d'euphonie, le français conserve, par exemple, le sigle anglais ASEAN: Association of Southeast Asian Nations, car la traduction en français de ce développement: Association des États d'Asie du Sud-Est, est ANASE, aux inflexions peu plaisantes. Autre exemple, l'acronyme français, AGETAC, pour «Accord général sur les tarifs douaniers et le commerce», équivalent du GATT recommandé par arrêté ministériel du 18 février 1987, ne s’est pas imposé. Le sigle anglais est donc conservé en français lorsque l'équivalent français manque d'euphonie.

Dans certaines circonstances, le sigle a un usage purement graphique, le manque d'euphonie tendant à imposer le recours au développement du sigle dans le discours oral. Ainsi, le sigle anglais HPAEs (Krugman et Obstfeld 1997: 265) étant difficile à prononcer, c'est le développement, High Performance Asian Economies, qui est utilisé le plus souvent en anglais. Nous noterons que le français n'emploie pas de traduction de ce sigle ou de son développement anglais. En revanche, nous avons constaté l'usage 
de la dénomination "économies dynamiques d'Asie» et du sigle correspondant, EDA, pour les mêmes pays et dans le même contexte (Perspectives économiques de l'OCDE 1999: 5).

La difficulté de prononciation présentée par IBRD, le sigle de l'International Bank for Reconstruction and Development, nous offre l'exemple d'un autre type d'adaptation. Ici le sigle cède la place à la dénomination consacrée par l'usage: World Bank. D'autre part, la traduction en français du développement du sigle, Banque Internationale pour la Reconstruction et le Développement, donne le sigle français $B I R D$ que beaucoup de français prononcent à l'anglaise comme le nom commun «bird»! Ou bien le français aura recours au sigle BM, pour Banque Mondiale. En définitive, lorsque le sigle est difficilement prononçable, le discours oral le remplace par son développement ou une dénomination simplificatrice consacrée par l'usage.

\section{I.2 Fonction Dénominative Lapidaire Véhiculaire (FDLV)}

La fonction dénominative lapidaire (FDL) peut être une fonction véhiculaire (FDLV). Sigles et acronymes évitent l'écriture, la lecture ou l'énonciation de longues suites de syntagmes. La FDLV va mettre des concepts obscurs à la portée de tous, les usagers de ces sigles grand public n'ayant, le plus souvent, plus aucun contact avec les formations lexicales composées exprimant les concepts. Bien peu d'acheteurs connaissent le sens de PAL/SECAM (Phase Alternating Line/Séquentiel à Mémoire). Peu leur importe de savoir que l'un de ces systèmes est allemand, l'autre français. Toutefois, c'est aux magnétoscopes PAL/SECAM que vont leurs préférences parce qu'ils savent que ce sont ceux qui offrent les meilleures performances. Nous ferons la même remarque pour le système NICAM: ce système est recherché car il dote les téléviseurs d'un son haute fidélité, numérique et stéréophonique; mais qui se soucie de savoir que NICAM est l'acronyme de Near Instantaneously Compounded Audio Multiplex? De même, les amateurs de mobilier de loisirs en PVC ne savent pas, pour la plupart, que ce matériau est du polychlorure de vinyle, le sigle étant le résultat de la réduction de la dénomination anglaise polyvinyl chloride. On pourrait ainsi multiplier les exemples.

La FDLV s'observe particulièrement dans les domaines techniques où la siglaison est très productive. Les termes étant généralement analytiques et descriptifs, les dénominations sont assez longues, ce qui justifie la réduction par le sigle (Depecker 1990: 141). Cette tendance, qui s'observe particulièrement dans le high-tech development, le développement des technologies de pointe - par exemple dans le domaine des nouvelles technologies de l'information et de la communication désigné par le sigle NTIC - a inspiré un acronyme, TechSpeak, et un livre à Edward Tenner: TechSpeak, or How to Talk High Tech (1989).

Cette FDLV est servie par le pouvoir évocateur particulier de certains acronymes, tels que ECU, SATELIT, SYNERGY. En effet, l'acronyme gagne en efficacité lorsqu'il conserve partiellement la dénotation d'origine de certains des termes de la composition initiale (Trouillon 1998: 218). Citons l'exemple de MERCOSUR, qui signifie Comision Sectorial para el Mercado Comun del Sur. Rares sont ceux qui, tout en étant capables de situer cet acronyme dans le contexte du commerce international, en connaissent le développement exact. Mais, pour l'étudiant français en économie, il n'est pas nécessaire d'avoir de grandes connaissances en langue espagnole pour distinguer 
mercado (marché) co = comun et sur (sud) dans MERCOSUR, acronyme qu'il utilisera couramment. S'il n'utilise pas l'acronyme, il parlera plutôt de «zone de libreéchange sud-américaine», dénomination qui constitue en fait une explication détaillée de la réalité représentée par MERCOSUR. En anglais, MERCOSUR se développe en Southern Cone Common Market, interprétation logique en langue anglaise d'un acronyme espagnol!

$\mathrm{Au}$ sein des institutions européennes, la communication écrite et orale n'échappe pas à la siglaison. La désignation des nouveaux programmes communautaires donne lieu à la formation de sigles et acronymes dont le choix se fait avec grand soin (Sarolea 1990 : 247). Outre les sigles et acronymes tels que nous les avons définis en introduction, la banque terminologique d'acronymes CORDIS entend par «acronymes» «les codes dont les éléments ne relèvent pas de mots (par exemple, SESAME)». C'est ainsi que les dénominations des programmes LINGUA, ERASMUS, SOCRATES ou TEMPUS, choisies pour leur pouvoir évocateur et/ou leurs connotations culturelles, sont assimilées à la catégorie des sigles et acronymes dont la formation originelle n'est plus présente à l'esprit des locuteurs, ceux-ci les situant simplement dans un contexte précis. D'ailleurs, qu'il soit représenté par un code suivant la définition du CORDIS ou par un sigle ou un acronyme issu de la contraction d'une formation syntaxique composée, le site Internet des programmes communautaires mentionne seulement la vocation de chaque programme, postulant sans doute que celle-ci importe davantage aux locuteurs que le développement éventuel de certains d'entre eux, tels qu'EUREKA, par exemple:

EUREKA, soutien à des projets de recherche;

LEADER, aide au développement rural (2000-2006);

LINGUA, promotion de l'apprentissage des langues (2000-2006);

SOCRATES, partenariat dans le domaine de l'éducation (2000-2006);

PESCA, aide à la restructuration de la pêche (1994-1999);

SYNERGY, aide au renforcement de la coopération énergétique internationale (1998-2002).

Peu importe si le sigle perd le contact précis avec la formation lexicale composée à l'origine de sa formation. L'essentiel est son ancrage dans le sociolecte d'une communauté linguistique ou d'un fragment de communauté, tel qu'un groupe socio-professionnel.

Le sigle créé pour les besoins d'un seul et unique document constitue enfin un cas particulier. Le chercheur qui rédige un article ou un ouvrage veut éviter la répétition fastidieuse d'une suite de syntagmes et pour ce faire propose un sigle. Il s'agira d'une convention entre le lecteur et lui. Ainsi pouvons-nous convenir de remplacer la collocation «sigles et acronymes» par S\&A. Par ailleurs, l'auteur peut espérer faire école.

\section{I.3 Fonction Dénominative Lapidaire Grégaire (FDLG) : les sigles pour initiés, du shop talk au show talk}

La FDL peut être une fonction grégaire (FDLG). Ici la compréhension est limitée à une communauté réduite. Le sigle peut même apparaître comme un procédé de cryptage lié à la préservation de la confidentialité ou du secret. Ces sigles pour initiés 
se trouvent dans certains domaines techniques, le secteur de la défense nationale, de la recherche dans des secteurs sensibles tels que le nucléaire. Les pratiques cryptiques sont aussi le propre de certaines communautés ésotériques.

Dans ce contexte, nous rencontrerons et pourront éventuellement employer le mot «jargon» en nous entourant de multiples précautions. Les hedging procedures sont ici fort utiles. En effet, déclare Walter Nash, «jargon is a species of crime against language and clear thinking» (1993: 3), un language à bannir! Cette précaution étant prise, Nash précise tous les aspects que peut prendre ce terme, par exemple pour qualifier la terminologie d'une profession, des spécialités techniques, ou pour couvrir le secret car "another sense [of jargon] is "secret language" » (1999: 4). C'est dans ce type d'acceptions que nous pouvons concevoir ici l'usage du mot "jargon».

Dans un domaine technique, les sigles pour initiés sont intelligibles uniquement pour les spécialistes. Certains sigles sont vulgarisés, portés à la connaissance et connus de tous, mais d'autres n'ont de sens que pour les initiés. Comme exemples de ces deux cas opposés, nous citerons «BU» et «ISBN». Le premier est un sigle grand public, réduction courante de la dénomination «bibliothèque universitaire». Quant au second, si certains lecteurs savent qu'il indique une référence de l'ouvrage concerné, bien peu d'entre eux en connaissent le développement, International Standard Book Number, une information dénuée d'intérêt pour la plupart. La justification du sigle est ici la commodité pratique: c'est ce que nous appellerons talking shop. Cela permet une communication plus rapide et plus efficace avec les initiés, the initiated. En revanche, the outsiders, or uninitiated, les non-initiés, ne comprendront pas, mais ce discours ne leur est pas destiné (Burke-Porter 1995).

Cette théorie du shop talking se combine à une seconde théorie, celle du secret, un moyen de communication que le public peut surprendre mais sera incapable de décoder. Le secret est justifié pour des raisons de sécurité, par exemple dans l'armée (Trouillon 1998) où les premiers sigles n'étaient guère plus que des mots-codes, en usage dans les états-majors militaires ou les services de renseignements. Le nucléaire, où l'atmosphère de secret qui a entouré les premières recherches a renforcé la siglomanie (Goffin 1989: 97), en est un autre exemple. Citons enfin les formules sigliques qu'affectionnent les sociétés d'initiés telles que les franc-maçons. En effet, les lettres font partie du symbolisme maçonnique avec des nombres et des formes telles que le triangle ou l'étoile à cinq branches. Cela n'est pas sans rapport avec nos remarques sur les domaines techniques, car à l'origine, au Moyen Âge, le savoir-faire des maçons et leurs secrets professionnels se transmettaient par cooptation et initiation afin de préserver le secret maçonnique. Les sociétés d'initiés sont d'ailleurs qualifiées de communautés ésotériques, ce dernier adjectif signifiant "réservé aux adeptes; peu compréhensible pour le commun des mortels; hermétique, obscur» (Larousse 1999).

Autre fonction d'une langue d'initiés: impressionner les non-initiés. Comme le linguiste Walter Nash l'a souligné, le shop talk est aussi show talk, un moyen d'impressionner les non initiés. Dans ce cas, le shop talk prend une membershipping function (Nash 1993: 98). Il permet d'affirmer ou de revendiquer son inclusion dans un groupe, dans divers styles de show talk. L'emploi d'un vocabulaire hermétique par un groupe social est en effet l'un des moyens les plus puissants d'inclusion et d'exclusion (Burke-Porter 1995: 13). Ainsi dans «Parlez-vous branché?», Barrera y Vidal (1989: 75-84) inclut-il la siglaison dans le "parler jeune», citant, en 1989, BCBG ou 
HS. Aujourd'hui ce "parler jeune» se caractérise essentiellement par le verlan qui permet aux jeunes de se reconnaître entre eux comme faisant partie d'une communauté.

\section{I.4 Fonction Dénominative Lapidaire Euphémique (FDLE)}

La fonction dénominative lapidaire (FDL) peut être une fonction euphémique (FDLE). «Expression atténuée d'une notion dont l'expression directe aurait quelque chose de déplaisant, de choquant» (Petit Robert 1999), l'euphémisme est un phénomène sociolinguistique dépendant du code moral et des interdits des sociétés concernées. Les euphémismes nous permettent d'éviter d'offenser les autres en respectant leurs droits (Hudson 1996: 114-115).

Le procédé euphémique consiste essentiellement en une substitution, un syntagme ou un groupe de syntagmes étant remplacé par un autre. Cette substitution s'effectue par le biais de divers moyens linguistiques, tels qu'antonyme négativé, métaphore, métonymie, emprunt ou périphrase (Tournier 1991: 70), parmi lesquels apparaît la réduction du signifiant par troncation ou siglaison. Ce dernier moyen peut s'illustrer, par exemple, par le sigle PWA: Person With AIDS ou, sigle moins brutal, PLWA, Person Living With AIDS. Avec cet exemple, nous abordons ici le discours médical dont l'euphémisation est une caractéristique permanente, en particulier dans la communication médecin-malade et malade-société. L'euphémisation est une fonction classique des sigles; leur usage vise à protéger autrui et à se protéger en minorant en quelque sorte la gravité ou l'intensité des faits. Les sigles peuvent aussi être utilisés pour coder l'inavouable, remarque Vidalenc (1999: 492); et l'on songera aux sigles médicaux totalement hermétiques inscrits sur les ordonnances de prélèvements sanguins pour analyses biologiques, tels que la recherche de certaines MST.

Claude Hagège qualifie ce procédé de «minoration euphémistique» dans laquelle «diverses ressources de la langue sont mises à profit pour refouler les sens et les images qui leur sont associés, en les camouflant sous la magie propitiatoire des noms biaisés» (1996: 259). Ces évolutions sémantiques peuvent être vues comme des marques de compassion, respect ou prévenance vis-à-vis d'autrui, ou bien, à l'opposé, comme l'expression de l'hypocrisie d'individus qui ne veulent surtout pas subir le coût d'une implication en dehors de leur sphère personnelle immédiate.

Tout ce qui est susceptible de choquer est banni du discours "politiquement correct», souci permanent de la communication actuelle. Le style de ce discours consiste à faire un usage abondant d'euphémismes; c'est une euphémisation de la langue. Perceptible dès les années 1980, c’est au début des années 1990 que cette tendance à une orthodoxie linguistique dure a été dénommée aux États Unis political correctness ou PC, le discours étant alors qualifié de politically correct, «politiquement correct» en français. En anglais, on peut noter depuis lors des collocations du type $P C$ activists, the $P C$ position, critics of $P C$, proponents of $P C$, the use of $P C$ terms, those who adopt a PC line, the PC frame of mind, politically correct ou political correctness ayant eux-même été réduits en sigle. En faisant abstraction de certaines dérives linguistiques obsessionnelles, nous noterons l'impact de cette tendance sur la terminologie d'un domaine spécialisé, la typologie du développement économique, par une approche comparative français/anglais. Ainsi la dénomination «pays sous-développés» a été bannie au profit des "pays en développement»; mais dans ce contexte, nous 
trouvons aussi divers sigles et développements de sigles dont nous allons esquisser les relations synchroniques et diachroniques.

Place des sigles dans la typologie des pays par niveau de développement

\begin{tabular}{|l|l|}
\hline $\begin{array}{l}\text { underdeveloped countries } \\
\text { LLDCs, least developed countries }\end{array}$ & $\begin{array}{l}\text { pays sous-développés* } \\
\text { PMA, pays les moins avancés }\end{array}$ \\
\hline $\begin{array}{l}\text { LDCs, less developed countries } \\
\text { developing countries }\end{array}$ & $\begin{array}{l}\text { PVD, pays en voie de développement } \\
\text { PED, pays en développement }\end{array}$ \\
\hline emerging countries/economies & pays/économies émergent(e)s \\
NICs, newly industrializing countries & NPI, nouveaux pays industrialisés/industriels \\
NICs, newly industrialized countries & NPI, nouveaux pays industrialisés \\
NIEs, newly industrializing economies & NEI, nouvelles économies industrialisées \\
NIEs, newly industrialized economies & NEI, nouvelles économies industrialisées \\
\hline industrial countries & pays industriels \\
industrialized countries & pays industrialisés \\
developed countries & pays développés \\
\hline
\end{tabular}

* Ces dénominations tendent à être évitées dans le discours "politiquement correct», leurs connotations étant jugées péjoratives.

Nous observons d'emblée que la siglaison ne s'impose pas avant une composition supérieure à deux syntagmes. Les dénominations sont figées dans les deux langues, et dans leurs équivalences. Si «pays développés» est l'équivalent de developed countries, LDCs: less developed countries ne se traduit pas mot-à-mot en français. Considéré comme synonyme de developing countries, son équivalent français est actuellement " pays en développement » (PED). En effet, on observe ici une évolution diachronique: "pays en voie de développement» tend à céder la place à la dénomination plus positive "pays en développement». Toutefois, le sigle français PVD persiste dans l'usage courant, la pratique n'ayant pas encore consacré le sigle PED. Nous avons relevé sur Internet: "les pays en développement (PVD)», signe que les nouveaux sigles ne s'imposent que progressivement, et que la vigilance est de rigueur dans le recours à l'information diffusée par Internet.

Le sigle $L L D C$ s et son développement least developed countries, utilisés par des auteurs spécialistes du domaine tels que Bela Belassa, se traduit par «PMA: pays les moins avancés». Toutefois, lors de la Conférence de la CNUCED à Bangkok en février 2000, les dépêches de l'AFP en anglais mentionnaient «least developed countries (LDCs)». Ayant adressé à l'AFP un mél relevant cette erreur, nous n'avons pas obtenu de réponse. Les finesses de la langue de spécialité échappent parfois aux plus avertis! En l'occurrence, il s'agit d'une inexactitude plus grave que le glissement opéré dans l'usage de PVD pour PED signalé ci-dessus, car LLDCs et LDCs font référence à deux catégories différentes de pays, ce qui n'était pas le cas de PVD et PED.

En ce qui concerne les pays émergents, l'équivalent du sigle français «NPI: nouveaux pays industrialisés / industriels », est sans conteste le sigle anglais NICs. Toutefois il existe une variation synchronique du développement du sigle anglais qui pourra être Newly Industrializing Countries ou Newly Industrialized Countries. Le contexte fera également référence à ces mêmes pays au moyen du sigle NIEs, développé soit en Newly Industrializing Economies, soit en Newly Industrialized Economies. La quasi- 
totalité des pages Internet sur le sujet emploient les deux libellés incluant les formes -izing, Newly Industrializing Countries et Newly Industrializing Economies. Toutefois, si l'on se réfère aux auteurs de deux bestsellers sur l'économie internationale, Krugman et Obstfeld (1997) emploient industrializing tandis que Dunn et Ingram (1999) emploient industrialized. Industrializing indique un processus en cours, newly, depuis peu; ce sont des pays dont l'industrialisation a commencé récemment. Industrialized est un constat de résultat mais sans précision du degré d'industrialisation. Ces divers éléments ne nous permettent donc pas de différencier vraiment les formes -izing et -ized dans ces dénominations, d'autant qu'elles qualifient les mêmes pays.

En conclusion, nous soulignerons l'impact déterminant du discours politiquement correct sur l'évolution de la terminologie de l'économie du développement et en particulier l'emploi des sigles dans ce contexte, l'objectif premier du recours au discours politiquement correct étant la valorisation des pays cités. La fonction euphémique des sigles et acronymes est donc une fonction véhiculaire minorée, infléchie par des considérations autres que la volonté de véhiculer un concept auprès du plus grand nombre.

\section{VIE DES SIGLES ET ACRONYMES}

Le choix, l'évolution et le succès des sigles et acronymes résultent d'un faisceau d'influences multiples: linguistiques, culturelles, économiques ou politiques. Nous soulignerons l'importance du rôle normatif des banques de termes ou services internationaux de terminologie pour la stabilisation des sigles et des développements correspondants (Sarolea 1990: 247).

\section{II.1 Variations synchroniques et diachroniques}

Nous évoquerons d'abord les variations synchroniques et diachroniques dans la transcription des sigles. Ces variations s'observent dans l'écriture en lettres capitales, avec ou sans ponctuation ou en minuscules. Sur le plan synchronique, Swan (1995: 2) considère que le British English moderne écrit les abréviations sans points tandis que ces mêmes points sont habituels en American English. Sur le plan synchronique et phonologique, et pour le cas abondamment documenté du sigle de l'Organisation des Nations unies (Mortureux 1995: 18), Lerat différencie l'épellation, à laquelle incite la graphie O.N.U., et la lecture syllabique, favorisée par la graphie ONU (1995: 58).

En règle générale, la transcription des sigles et acronymes subit l'évolution diachronique suivante: un sigle commence par s'écrire avec des capitales séparées par des points. Ensuite, par étapes successives, ces points disparaissent et des capitales ne subsiste que l'initiale (pour une étude détaillée de ces variations, voir Losson 1990). Enfin, à l'exception des sigles ou acronymes noms propres, si leur usage s'intensifie, ils pourront finir par s'écrire totalement en minuscules, et, forme ultime de la lexicalisation, devenir un mot comme un autre qu'il faudra accorder (un laser, des lasers, un radar, des radars) et servir de base de dérivation.

Considérons le cas de l'ECU. En 1989, Jean-Paul Mingasson (1989: 65), président de l'Ecu Interservice Group, signale que la Commission des Communautés européennes emploie «ECU» en anglais et «l'Ecu» en français. «These written forms lay 
stress on its derivation from European Currency Unit, giving the ecu the air of an artificially constructed unit of account». Le groupe qu'il représente préconise les formes «ecu» en anglais et "l'écu» en français, sans majuscules, dans un but d'harmonisation avec les autres monnaies «ecu, dollar, sterling, franc, yen», et surtout "pronounced as in French, not "EE-SEE-YOU" (voir aussi en II.2 une explication de cette exigence phonétique). L'assimilation graphique et phonétique à l'ancienne monnaie française permet au sigle de brûler les étapes vers la lexicalisation, la prononciation à la française donnant à «ecu» en anglais une valeur d'emprunt.

Le choix des sigles et des acronymes peut également être sujet à des variations synchroniques. Leur choix est fortement influencé par les origines socioculturelles des locuteurs, par exemple l'appartenance à un groupe socio-professionnel (membershipping function), comme en témoigne l'illustration suivante prise dans le secteur associatif, terrain fertile pour les créations d'acronymes. Voici un passage révélateur tiré d'un article de la revue Problèmes économiques, citant «Des associations entendues au sens large: en espéranto bruxellois, le CMAF (coopératives, mutuelles, associations et fondations), en jargon d'experts, le SBNL (secteur à but non lucratif) ou encore le "tiers secteur" en dialecte sociologique» (Malaurie 1999: 22-23). "Espéranto", "jargon» et "dialecte» sont des termes éloquents. Chacun de ces termes qualifie une communauté de langage plus ou moins étendue: l'espéranto, c'est la langue internationale, le jargon est ici l'idiome que se fabrique une communauté spécialisée afin de communiquer de façon plus ou moins hermétique, tandis que «dialecte» est pris au sens de dialecte social, c'est-à-dire un ensemble de termes utilisés dans un groupe social.

\section{II.2 Changement de sigle}

"La longévité d'un néologisme est imprévisible, mais liée au besoin du terme, à la durée du référent, à sa dissémination, à la défaite des termes concurrentiels, et à la richesse du réseau d'associations qui l'enserre» (Forgue-McDavid 1972: 156). Néologismes formés par réduction morphologique, les sigles et les acronymes peuvent donc être remplacés, modifiés ou changer de développement, en fonction de la vie de leurs référents et pour des raisons historiques, juridiques, sociolinguistiques aussi bien que psycholinguistiques.

Un sigle peut être remplacé par un autre sigle lorsque les caractéristiques du référent sont modifiées. La modification du signifiant est alors effectuée pour maintenir l'adéquation avec le signifié. Les exemples abondent; nous citerons le cas de la CEE, EEC ou European Economic Community remplacée par l'UE, l'Union Européenne, EU ou European Union. Le GATT a été remplacé par le WTO; mais alors que GATT n'avait pas été traduit en français, WTO, World Trade Organization a donné en français OMC, Organisation Mondiale du Commerce. En fait il s'agit de deux entités différentes; schématiquement, nous dirons que le GATT était un accord tandis que l'OMC est une institution. Ces changements de sigles ont été motivés par d'importantes transformations structurelles.

C'est parfois l'interaction de plusieurs motifs qui va occasionner le changement de sigle. Pour illustrer ce cas, évoquons un destin fugace, celui de l'écu, European Currency Unit, dont Losson (1990: 33) soulignait, en 1990, le pouvoir évocateur « appelé à avoir un bel avenir tout en renouant sous ce nom, du moins pour le français, 
sentimentalement avec le passé». C’est un exemple de sigle ou acronyme motivé, c'est-à-dire une forme dont la désignation est en rapport avec le sens de son homonyme. L'écu a été remplacé par l'euro et, en tant que sigle de l'unité monétaire européenne, appartient à l'histoire.

Selon Krugman et Obstfeld (1997: 517), le fait que l'écu soit le nom d'une ancienne pièce d'argent française n'était pas une coïncidence. Depuis 1979, un panier de devises européennes était appelé «the European Currency Unit (ECU)». Le terme «Euro» fut choisi en décembre 1995. Dans le traité de Maastricht, la monnaie unique est appelée ECU, mais la plupart des dirigeants européens n'approuvaient pas l'adoption du nom d'un panier de devises préexistant, lequel s'était, en outre, fortement déprécié par rapport au Deutsch Mark. De plus, le Chancelier allemand Kohl objectait qu'en allemand «ein ECU» se prononce comme "eine Kuh», une vache (Krugman et Obstfeld 1997: 640). Cet ensemble de raisons économiques, politiques et phonétiques signe la défaite de l'écu et, «[e]n janvier 1999, l'ECU cède le pas à l'euro. Un sigle se voit remplacé par un fractomorphème nouvellement affranchi» (Humbley 1999: 169).

Dans le contexte de l'économie internationale, les HIPCs (Highly Indebted Poor Countries), que nous avons évoqués plus haut, nous permettent de mentionner un dernier cas de changement de sigle. Cette catégorie créée en 1996 remplaçait les SILICs: Severely Indebted Low Income Countries; « une dramatisation sémantique a transformé la catégorie "technique" utilisée jusque là (Severely Indebted Low Income Countries) en une nouvelle catégorie de pauvres (les Highly Indebted Poor Countries ou HIPCs, PPTE en français)», soulignent Moisseron et Raffinot (1999: 36). Quant à nous, nous ne voyons dans le remplacement des caractéristiques «severely indebted low income» par "highly indebted poor» qu'un recours délibéré au langage "politiquement correct» dont l'incidence nous paraît très minime.

Un changement de sigle peut donc être la conséquence d'une motivation unique mais résulte le plus souvent d'un ensemble de raisons très diverses se renforçant entre elles.

\section{II.3 Modification du développement du sigle}

Certaines abréviations manifestent une plus grande stabilité que leurs développements; elles acquièrent une existence propre (Nkwenti-Sager 1990: 100). Tel est le cas de l'acronyme de l'UNICEF créé en décembre 1946 en tant que United Nations International Children's Emergency Fund, fonds destiné à porter secours aux enfants des pays dévastés par la guerre. Après 1950, quand l'urgence de l'après-guerre fut passée, les efforts du Fonds furent orientés vers des programmes d'amélioration du bien-être des enfants, en particulier dans les pays en développement ainsi que dans des situations diverses d'urgence. L'UNICEF devint alors United Nations Children's Fund, le changement de nom voulant refléter ce changement de fonction (Encyclopadia Britannica, Micropadia, $15^{\mathrm{e}}$ éd., vol. X, p. 268). On observe donc ici une variation diachronique du développement pour un acronyme inchangé. Si le développement a été modifié pour des raisons conjoncturelles, le sigle initial, de réputation internationale, a toutefois été conservé.

Ce sont d'autres raisons qui ont motivé la variation diachronique du développement des acronymes MATIF et LIFFE. Le MATIF était à l'origine le «marché à terme 
des instruments financiers». Le sigle correspondait alors parfaitement à son développement. Ce dernier a changé pour devenir «marché à terme international de France» dans un effort d'harmonisation avec des marchés concurrents à l'étranger, tels que le LIFFE à Londres et le NYMEX à New York, dont les acronymes indiquent la localisation. L'acronyme MATIF reste inchangé pour un même nombre de mots contenus dans le développement, mais certains de ces mots sont nouveaux. De nouveaux éléments techniques, les options, sont inclus dans le MATIF mais ils ne figurent pas dans le développement du sigle contrairement au développement du sigle anglais de LIFFE. En effet, le LIFFE, London International Financial Futures, marché à terme d'instruments financiers créé en 1982, est devenu en 1992 le London International Financial Futures and Options Exchange, marché à terme d'instruments financiers et d'options de Londres, après sa fusion avec le London Traded Option Market (LTOM), marché des options créé en 1978 (Esposito 1997). L'acronyme reste inchangé alors que le développement est augmenté pour tenir compte d'éléments techniques nouveaux, les options. Pour le MATIF comme pour le LIFFE, l'acronyme est resté inchangé alors que le développement était modifié pour des raisons différentes, raisons de politique économique ou raisons techniques.

Enfin, dans un dernier exemple, nous ferons un constat de variation synchronique du développement d'un acronyme, et signalerons la variation diachronique hypothétique envisagée par The Economist. L'acronyme NAFTA, North-American Free Trade Agreement, se retrouve parfois développé en North-American Free Trade Area. Dans leurs ouvrages respectifs publiés récemment, Krugman et Obstfeld (1997) n'emploient que le développement incluant agreement, alors que Dunn et Ingram (1999) utilisent les deux possibilités de développement: le corps du livre mentionne seulement agreement $(180,189)$ - ainsi dans «the fight over the passage of NAFTA » (118), la collocation "passage of NAFTA» implique nécessairement «the passage of an agreement» - alors que le glossaire en fin d'ouvrage (547) indique seulement que «NAFTA is a free trade area consisting of the US, Canada and Mexico». Par ailleurs, The Economist (15 avril 2000, p. 36) évoque des perspectives d'évolution du développement de l'acronyme: si «The idea that Britain could join the North American Free Trade Agreement is dismissed as a fantasy of anti-European Tories [...] the enthusiasts point out that the NAFTA acronym could easily be adapted to stand for North Atlantic». Cela appelle deux remarques. La modification du développement, avec substitution d'area à agreement, s'effectue par cohérence avec un concept qui constitue une collocation d'importance majeure dans le contexte du commerce international: free trade area (d'autant que NAFTA est une zone de libreéchange). D'autre part, le remplacement éventuel de North-American par NorthAtlantic, s'il ne pose aucun problème linguistique car dans les deux cas il s'agit d'un adjectif composé, serait conditionné par une nouvelle adhésion à l'accord, adhésion dépendant de décisions de politique économique internationale.

La modification du développement des sigles peut donc s'effectuer en maintenant totalement la relation avec les initiales d'origine (MATIF, NAFTA) ou bien en ne conservant que partiellement ce contact (UNICEF, LIFFE). Elle peut être due à des raisons techniques ou conjoncturelles, de politique économique intérieure ou internationale. 


\section{II.4 Conditions de succès ou d'altération des performances}

Au-delà de trois lettres, les sigles épelés ont parfois quelque difficulté à s'imposer, difficulté liée au problème de prononciation et de mémorisation. En revanche, les acronymes se retiennent facilement et s'imposent plus vite. La perception de la motivation est évidemment tributaire de la culture des locuteurs. C'est leur culture qui leur fait particulièrement apprécier les personnages de la mythologie (IRIS, PANDORA), de la littérature: DANTE (Delivery of Advanced Network Technology to Europe limited), les termes latins et grecs (ERASMUS, EUREKA).

L'importance de la qualité attractive du sigle ou de l'acronyme est liée, par exemple, à la nécessité de séduire une clientèle ou de retenir l'attention internationale. Dans les conditions optimales, les sigles et acronymes doivent être faciles à retenir, attirants et parlants. L'aspect ludique des termes créés est un gage de facilité d'intégration dans le langage. Pour qu'il soit plus facile à retenir, il faut chercher un acronyme, homonyme de mots existants, «dont le sens motive (surdétermine) celui du sigle ou acronyme» (Mortureux 1997: 54); ce mot pourra apporter à l'acronyme des éléments favorables supplémentaires par ses propres dénotations, comme, par exemple, INSIGHT: Information System Integrated by using Global Hypermedia Technology, EASI: European Academic Supercomputing Initiative, ou EARN: European Academic and Research Network (site Inforoutes).

Certains sigles sont le produit de la fantaisie (Goffin 1989: 97). Nous citerons certains S\&A particulièrement ludiques : Etcetera, RAPIDE, STENO, CHIC... Etcetera ne pose aucun problème de mémorisation; on ne peut en dire autant de son développement East-West Technical Cooperation in the Research and Development of Electronic Trading (site Inforoutes).

Si une partie au moins de l'acronyme peut donner une idée de ce qu'il représente ou de son contexte d'implantation, l'usage et la mémorisation en sont facilités; c'est le cas pour INMARSAT (International Maritime Satellite Organisation) ou SATELIT (Société Autonome des Télécommunications du Togo) (cf. Inforoutes) ou MEDICA, Multimedia Medical Diagnostic Assistant.

Les acronymes prennent parfois l'apparence d'un homonyme de vocable anglais pour un développement français. On trouve, par exemple, CALF (Centre d'Apprentissage de la Langue Française) ou bien, à la pointe de l'actualité française: CARE, le Contrat d'Aide pour le Retour à l'Emploi. Souhaiterait-on ainsi faire jouer la fascination qu'exerce tout terme anglais sur nombre de francophones pour garantir le succès du nouvel acronyme? Mais il existe aussi des acronymes de consonance française pour un développement anglais. ECLAIR, European Collaborative Linkage of Agriculture and Industry through Research, et FLAIR, Food-Linked Agro-Industrial Research, en sont deux exemples. Dans les deux cas, nous concluerons que l'objectif poursuivi est avant tout l'homonymie avec un terme existant dont le sémantisme doit renforcer le signifié du sigle ou de l'acronyme (voir aussi Clas 1990: 151). À cet objectif s'ajoute alors un attrait supplémentaire, l'aspect exotique de l'emprunt.

En revanche, l'existence de sigles et acronymes homonymes (mots écrits et prononcés de façon identique) est un problème susceptible d'altérer leurs fonctions. En économie, $P P P$ se développe en purchasing power parity, polluter pays principle (le principe du pollueur payeur en économie de l'environnement), pool purchase price (en économie de l'énergie), mais aussi Protocole Point à Point/Point to Point Protocol 
(cité par les Inforoutes de l'espace francophone). LSE peut se développer en London Stock Exchange mais aussi en London School of Economics. Et les banques de données terminologiques telles que CORDIS ou EURODICAUTOM révèlent l'existence d'homonymes multiples pour de nombreux sigles et acronymes. D'où l'importance de l'évaluation du contexte concerné (Percebois 1999: 239-241 et 244-245). Kwenti et Sager proposent une solution à ce problème: l'utilisation de conventions typographiques différentes, par exemple Btu pour British Thermal Unit, et B.T.U. pour Board of Trade Unit. Ces termes sont alors toujours homophones (même prononciation, sens et/ou orthographe différents) mais non homographes (orthographe commune, sens et/ou prononciation différents). À ce sujet, nous signalerons l'exemple des précautions similaires prises pour les transcriptions de textes relatifs au cycle nucléaire afin de bien différencier Am: l'americium et AM: les actinides mineurs. Si le procédé préconisé par Kwenti Sager semble être un moyen très utile à l'avenir pour prévenir les cas d'homographie, il ne résoud pas le problème d'homophonie qui nécessitera davantage de précisions à l'oral. Ces remarques soulignent l'intérêt d'une vérification préalable de l'existence d'homonymes lors de la création d'un nouveau sigle ou d'un nouvel acronyme.

\section{II.5 Conséquence du succès : la lexicalisation}

La lexicalisation marque la coupure sémantique entre le sigle et sa source. "Le sigle est en cela intégré au système sémiologique plus large qu'est la langue. Il n'est plus une abréviation, renvoyant sans arrêt à son origine, il est une unité du système» (Calvet 1980 : 119). Mots à part entière, sigles et acronymes vont s'accorder, la marque du pluriel étant le premier signe de cette évolution.

La lexicalisation des sigles s'affirme ensuite dans la dérivation par l'adjonction de suffixes; c'est la consécration pour le sigle ou l'acronyme. Devenant une base de dérivation suffixale, le sigle fonctionne comme un nom, sujet à transformations: transformation adjectivale, la plus fréquente, qui donne «smicard, cégétiste, onusien, capésien, énarque », mais aussi transformation verbale. Nous rappellerons à cet égard l'exemple cité par Vidalenc (1999: 492) dans le domaine médical. Dx, étant au départ le diagnostic, devient verbe régulier ( «I was Dx'd in 6/93 with a bPSA of 2.8 ») ou irrégulier ( «when I was Dx for Pca»). Dans le contexte économique, un exemple tout récent montre la complexité de certaines compositions. The Economist (13 mai 2000 p. 78) titre: «Ecofinessed». Réduisant irrévérencieusement à « Ecofin » la dénomination de l'Economic and Financial Council, institution de l'Union européenne, le journaliste élargit ensuite l'usage de nom de cet acronyme à celui de verbe au participe passé: «ecofinessed». C'est un procédé néologique au service d'une intention très pointue du rédacteur ou du locuteur qui adapte parfaitement sa terminologie au contexte plutôt que d'avoir recours à un terme banal d'usage courant. Ce procédé met en valeur le talent ou même la virtuosité verbale de l'auteur. En titre, comme dans cet article, ce terme intrigue et incite à lire l'article pour trouver la clé permettant son interprétation. 


\section{CONCLUSION}

En réponse à un besoin de communication, d'économie linguistique et d'euphonie, sigles et acronymes ont donc une fonction fondamentale : la FDL, Fonction Dénominative Lapidaire. En outre, cette fonction peut être Véhiculaire (FDLV), Grégaire (FDLG) avec les sigles pour initiés dans des usages diversifiés du shop-talk au showtalk, ou encore Euphémique (FDLE), fonction dont les caractéristiques sont susceptibles de s'apparenter aussi bien à celles de la fonction véhiculaire que de la fonction grégaire.

L'observation de la vie des sigles et acronymes fait apparaître de nombreuses variations synchroniques et diachroniques dans leur usage. Elle permet également de dégager les raisons des changements de sigle ou des modifications du développement des S\&A et de distinguer certaines conditions de succès ou d'altération de leurs performances, telles que l'homonymie. Enfin, sigles et acronymes parviennent au stade ultime de leur évolution avec leur complète lexicalisation.

Si l'impératif de clarté recommande parfois le recours aux formes abrégées, ce même impératif peut aussi s'y opposer. L'analyse comparée anglais-français permet d'ailleurs de constater qu'un sigle français n'est pas nécessairement rendu par un sigle anglais et inversement, la préférence du locuteur pouvant aller à l'emploi du développement pour des raisons de facilité ou difficulté de prononciation du sigle, ou pour la recherche de précision et d'insistance sur une composante particulière non évidente dans le sigle; par exemple, on aura recours au développement du LIFFE pour insister particulièrement sur le marché des options.

La création des sigles et acronymes n'est pas un phénomène isolé, mais résulte de la rencontre et de la pression de forces linguistiques, sociolinguistiques et psycholinguistiques. Si leur apparition massive témoigne de la vitalité créative de l'écrit, leur forme graphique est souvent inspirée par la possibilité de leur réalisation orale, qui est souvent déterminante pour leur longévité. Par ce procédé, la formulation des concepts gagne en concision et en impact. Si l'on a pu croire, passagèrement, et sur un constat superficiel, à une mode, les services rendus par les sigles et acronymes en ont fait un mode d'expression irremplaçable dans tous les domaines d'activité ou de spécialité.

\section{RÉFÉRENCES}

Barrera y Vidal, A. (1989): «Parlez-vous branché?», dans Terminologie et traduction, n 1 , p. 75-84.

Burke, P. and r. Porter (1995): Languages and Jargons, Cambridge, USA, Polity Press, Backwell. Calvet, J.-L. (1980): Les sigles, Paris, PUF.

CAlvet, J.-L. (1999) : L'enquête sociolinguistique, Paris, L'Harmattan.

Clas, A. (1990) : «Brachygraphie et oralité», dans Terminologie et traduction n ${ }^{\circ} 2$, p. 149-156.

DeAK, E. et S. (1981): Grand Dictionnaire d'américanismes, Paris, Éditions du Dauphin.

Depecker, L. (1990): "Réflexion sur la question des sigles dans la normalisation», dans Terminologie et traduction, $\mathrm{n}^{\circ}$ 2, p. 141-146.

Dunn, R. and J. Ingram (1999): International Economics, printed in the United States, John Wiley \& Sons.

Esposito, M.-C. (1997) : Dictionnaire de l'économie, de la finance et de la comptabilité (anglais/ français et français/anglais), Paris, Economica.

Forgue, G. J. et Mcdavid JR., R.I. (1972): La langue des Américains, Paris, Aubier Montaigne. 
Goffin, R (1989): «La terminologie des sciences et des techniques nucléaires. Un cas de diachronie récente», dans Terminologie diachronique, Centre de terminologie de Bruxelles, conseil International de la langue française, p. 94-107.

Gramley, S. and K.-M. Patzold (1992): A Survey of Modern English, London, Routledge.

Grevisse, M. (1975): Le bon usage, imprimé en Belgique, Duculot.

Guilbert, L. (1975): La créativité lexicale, Paris, Larousse.

Hagege, C. (1996): L'homme de paroles. Contribution linguistique aux sciences humaines, Paris, Fayard.

Humbley, J. (1999): "Postface», dans Regards linguistiques et culturels sur l'euro, Rosalind Greenstein (éd.), Paris, L'Harmattan, p. 169-171.

Krugman, P. and M. Obstfeld (1997): International Economics, printed in the United States, Addison-Wesley.

Lerat, P. (1995): Les langues spécialisées, Paris, PUF.

Losson, G. (1990): «De l'emploi des formes abrégées dans les actes normatifs des institutions de la Communauté européenne», dans Terminologie et traduction, $\mathrm{n}^{\circ}$ 2, p. 7-34.

Malaurie, G. (1999) : «Le boom des Associations", dans Problèmes économiques, n 2.605, p. 22-26.

Mingasson, J.-P. (1989): “The written form of 'ECU', dans Terminologie et traduction, $\mathrm{n}^{\circ} 1$, p. 65-66.

Moisseron, J.-Y. et M. Raffinot (1999): Dette et pauvreté. Solvabilité et allègement de la dette des pays à faible revenu, Paris, Economica.

Mortureux, M.-F. (1994) : «Siglaison-acronymie et néologie lexicale», dans LINX, n 30, 11-32.

Mortureux, M.-F. (1995): "Les vocabulaires scientifiques et techniques», dans Les enjeux des discours spécialisés, Les Carnets du Cediscor, 3, Presses de la Sorbonne nouvelle, p. 13-25.

Mortureux, M.-F. (1997): La lexicologie entre langue et discours, Paris, SEDES.

NAsh, W. (1993): Jargon, Its Uses and Abuses, Oxford, Blackwell.

Nkwenti-Azeh, B. and J. C. Sager (1990): "Abbreviated forms in English", dans Terminologie et traduction, $\mathrm{n}^{\circ}$ 2, p. 93-101.

Partridge, E. (1973): Usage \& Abusage, printed in Great Britain, Penguin Reference Books.

Percebois, J. (1999) : «L'Interconnector: Un réseau de transmission de gaz européen. Approche lexicologique comparative du français et de l'anglais des réseaux et contrats gaziers ", dans ASp, 23/26, p. 223-252.

Perspectives économiques de l'OCDE (1999): «La situation économique des grandes régions du monde», dans Problèmes économiques $\mathrm{n}^{\circ} 2.632,22-09$, p. 1-6.

Rey-Debove, J. (1998): La linguistique du signe, Paris, Armand Colin.

Sarolea, R. (1990): «Les accrocs des acronymes», dans Terminologie et Traduction, n 2, p. 247251.

Swan, M. (1995): Practical English Usage, Oxford, Oxford University Press.

Tenner, E. (1989): TechSpeak, or How to Talk High Tech, London, Kogan Page.

Tournier, J. (1991): Structures lexicales de l'anglais, Paris, Nathan.

Tournier, J. (1993): Précis de lexicologie anglaise, Paris, Nathan.

Trouillon, J.-L. (1998): «Langues de spécialité et noms propres: matériels militaires», dans ASp, 19/22, p. 209-225.

Vidalenc, J.-L. (1999): "Faisabilité d'un enseignement à distance de phénomènes lexicaux et syntaxiques», dans ASp, 23/26, p. 483-500.

Widdowson, H. G. (1996) : Linguistics, Oxford, Oxford University Press.

\section{Dictionnaires}

Larousse 1999. Petit Robert, 1999.

Littré, É. Dictionnaire de la langue française.

\section{Sites Web}

Programmes communautaires de $\mathrm{A}$ à $\mathrm{Z}$

Banque de données CORDIS

Les Inforoutes dans l'espace francophone

$<$ http://www.tpfrance.org/cec/homecec.htm $>$ $<$ http ://www.cordis.lu/fr/src/d_002_fr.htm $>$ $<$ http ://inforoutes.cidif.org/sigles/> 
Développement des sigles et acronymes français cités

$\begin{array}{ll}\text { AGETAC } & \text { Accord Général sur les Tarifs douaniers et le Commerce (GATT) } \\ \text { ALENA } & \text { Accord de Libre Échange Nord-Américain (NAFTA) } \\ \text { ANASE } & \text { Association des Nations d'Asie du Sud-Est (ASEAN) } \\ \text { BIRD } & \text { Banque Internationale pour la Reconstruction et le Développement } \\ & \text { (IBRD) } \\ \text { CALF } & \text { Centre d'Apprentissage de la Langue Française } \\ \text { CARE } & \text { Contrat d'Aide pour le Retour à l'Emploi } \\ \text { CEE } & \text { Communauté Économique Européenne (EEC) } \\ \text { CNUCED } & \text { Commission des Nations Unies sur le Commerce et le Développement } \\ & \text { (UNCTAD) } \\ \text { EURODICAUTOM } & \text { Banque de données terminologique de la Commission } \\ & \text { (Europe dictionnaire automatique) } \\ \text { FMI } & \text { Fonds Monétaire International (IMF) } \\ \text { LEADER } & \text { Liaison Entre Actions de Développement de l'Economie Rurale } \\ \text { MATIF } & \text { Marché À Terme International de France } \\ \text { NPI } & \text { Nouveaux Pays Industrialisés (NICs) } \\ \text { NTIC } & \text { Nouvelles Technologies de l'Information et de la Communication } \\ \text { OIT } & \text { Organisation Internationale du Travail (ILO) } \\ \text { OMC } & \text { Organisation Mondiale du Commerce (WTO) } \\ \text { ONG } & \text { Organisation Non Gouvernementale } \\ \text { PED } & \text { Pays En Développement } \\ \text { PMA } & \text { Pays les Moins Avancés } \\ \text { PPTE } & \text { Pays Pauvres Très Endettés (HIPC) } \\ \text { RAPIDE } & \text { Réseau Africain pour l'Intégration et le Développement } \\ \text { SATELIT } & \text { Société Autonome des Télécommunications du Togo } \\ \text { SBNL } & \text { Secteur à But Non Lucratif } \\ \text { UME } & \text { Unité Monétaire Européenne } \\ \text { UE } & \text { Union Européenne (EU) }\end{array}$

Développement des sigles et acronymes anglais cités

$\begin{array}{ll}\text { ASEAN } & \text { Association of Southeast Asian Nations (ANASE) } \\ \text { CHIC } & \text { Constraint Handling in Industry and Commerce } \\ \text { DANTE } & \text { Delivery of Advanced Network Technology to Europe limited } \\ \text { EARN } & \text { European Academic and Research Network } \\ \text { EASI } & \text { European Academic Supercomputing Initiative } \\ \text { ECU } & \text { European Currency Unit (UME) } \\ \text { EEC } & \text { European Economic Community (CEE) } \\ \text { ERASMUS } & \text { European Community Action Scheme for the Mobility of University } \\ & \text { Students } \\ \text { Etcetera } & \text { East-West Technical Cooperation in the research and development of } \\ & \text { electronic trading } \\ \text { EU } & \text { European Union (UE) } \\ \text { EUREKA } & \text { European Research Coordination Agency } \\ \text { GATT } & \text { General Agreement on Tariffs and Trade (AGETAC) } \\ \text { HIPC } & \text { Heavily Indebted Poor Countries (PPTE) } \\ \text { HPAES } & \text { High Performance Asian Economies } \\ \text { IBRD } & \text { International Bank for Reconstruction and Development (BIRD) } \\ \text { ILO } & \text { International Labour Organization (OIT) } \\ \text { IMF } & \text { International Monetary Fund (FMI) } \\ \text { INMARSAT } & \text { International Maritime Satellite Organisation }\end{array}$


INSIGHT

IRS

LDCs

LIFFE

LLDCs

LSE

MEDICA

NYMEX

NYSE

NAFTA

NGOs

NICs

PANDORA

PLWA

PWA

QWANGOs

STENO

TRIPS

UNICEF

UNCTAD

WTO
Information System Integrated by using Global Hypermedia Technology

Internal Revenue Service (US) / Inland Revenue Service (UK)

Less Developed Countries

London International Financial Futures and Options Exchange

Least Developed Countries (PMA)

London School of Economics / London Stock Exchange

Multimedia Medical Diagnostic Assistant.

New York Mercantile Exchange

New York Stock Exchange

North-American Free Trade Agreement (ALENA)

Non Governmental Organizations (ONG)

Newly Industrializing Countries (NPI)

Prototyping A Navigation Database of Road-Network Attributes (projet du programme européen DRIVE 1)

Person Living With Aids

Person With Aids

Quasi Autonomous Non Governmental Organisations

Science, Technology, Environment Organization

Trade Related Aspects of Intellectual Property Rights

United Nations Children's Fund

United Nations Commission on Trade and Development (CNUCED)

World Trade Organisation (OMC) 